Revista de Psicología Vol. 29 (2), 2011 (ISSN 0254-9247)

\title{
Escala de Bienestar Subjetivo en Cuidadores Familiares de Adultos Mayores (EBEMS/CFAM) ${ }^{1}$
}

\author{
Miriam Teresa Domínguez Guedea ${ }^{2}$, María Fernanda Mandujano Jáquez ${ }^{3}$, \\ Germán López Dávalos ${ }^{4}$, Rosario Leticia Domínguez Guedea ${ }^{5}$, Manuel Jorge \\ González Montesinos ${ }^{6}$, Marcela Sotomayor Petearson ${ }^{7}$ y Blanca Fraijo Sing ${ }^{8}$ \\ Universidad de Sonora, México
}

El bienestar es un concepto que ha despertado un interés multidisciplinar y particularmente en la línea de estudios sobre cuidadores familiares de adultos mayores se ha registrado la necesidad de analizar dicho atributo ante el inminente aumento de familias cuidadoras. El objetivo de este trabajo es describir el proceso de diseńo, validación exploratoria y confirmatoria, así como la utilidad actual de la Escala de Bienestar Subjetivo para Cuidadores Familiares de Adultos Mayores - EBEMS/CFAM. Se presenta una secuencia de resultados de análisis cualitativos, análisis factoriales, de modelamiento Rasch y ecuaciones estructurales en cuatro muestras de estudio que, en conjunto, confirman la validez y confiabilidad de la escala.

Palabras clave: bienestar, cuidadores-familiares, adultos-mayores, validación, escala.

\section{Subjective Well-Being Scale of Family Caregivers for Older Adults (EBEMS/CFAM)}

Well-being is a concept that has aroused multidisciplinary interest. Particularly in studies about family caregivers of older adults there has been the need to analyze this attribute facing the imminent increase of family caregivers. The goal of this paper is to describe the design process, the exploratory and confirmatory validation, as well as the usefulness of the current Scale of Subjective Well-Being of Family Caregivers for Older Adults - EBEMS/ CFAM. Results are shown in a sequence, from qualitative analysis, to factor analysis, Rasch modeling and structural equations in four samples of study that, taken together, confirm the validity and reliability of the scale.

Keywords: Well-being, family caregivers, elders, validation, scale. 

Entendido como un fin o como proceso, el concepto del bienestar ha captado la atención desde diferentes abordajes, ha movilizado discusiones y propuestas de la psicología positiva, ha revolucionado perspectivas de análisis para la salud mental, ha suscitado discusiones que replantean el papel de lo subjetivo y lo material para alcanzar la felicidad y, con toda una larga historia de aproximaciones para su entendimiento, goza de un protagonismo en el marco actual de la política pública. Pero, ¿Cómo se traduce un concepto que tiene raíces epistemológicas complejas que van del abordaje individual al social? ¿Cómo se operacionaliza el bienestar? ¿Qué se entiende por felicidad?

Desde una perspectiva social se puede hablar de la existencia de un estado de bienestar cuando este asegura la protección social en términos de los derechos a la sanidad, vivienda, educación, seguro de desempleo y las pensiones de jubilación a los ciudadanos y, en este sentido, la "política social, concebida como desarrollo humano, está basada en el

1 Proyecto 99094 "Evaluación de un modelo estructural de bienestar subjetivo en cuidadores familiares de adultos mayores", financiado por el Fondo Sectorial Sectorial para la Educación SEP-CONACYT.

2 Doctora en Psicología, se dedica a la investigación básica y aplicada sobre salud y bienestar en familias con miembros adultos mayores. Pertenece al Sistema Nacional de Investigadores, Líder del Cuerpo Académico "Salud comunitaria y promoción de la salud en grupos vulnerables". Contacto: Departamento de Psicología y Cs. de la Comunicación, Universidad de Sonora, Blvd. Luis Encinas y Rosales s/n, CP 83000, Colonia Centro, Hermosillo, Sonora; miriamd@sociales.uson.mx

3 Licenciada en Psicología, cursando la Maestría en el Posgrado Integral de Ciencias Sociales de la Universidad de Sonora. Contacto: maferm@hotmail.com

4 Maestro en políticas y gestión del desarrollo social. Contacto: gld_first@hotmail.com

5 Maestra en innovación educativa, se dedica a la investigación sobre evaluación educativa y desarrollo de instrumentos psicométricos. Contacto: ldominguez@psicom.uson.mx

6 Doctor en Psicología Educativa con especialidad en Medición y Metodología en Ciencias Sociales. Investigador psicómetra del Instituto Nacional para la Evaluación de la Educación (INEE). Contacto: mgm@caborca.uson.mx

7 Doctora en Psicología. Se dedica a la investigación sobre salud y familia. Contacto: msotomayor@psicom.uson.mx

8 Doctora en Ciencias Sociales. Se dedica a la investigación ambiental, procesos educativos y desarrollo de instrumentos psicométricos. Contacto: bfraijo@sociales.uson.mx 
enfoque contemporáneo del bienestar y desarrollo social" (Draibe \& Riesco, 2009, p. 61). Pero en esta misma perspectiva se reconoce que, además de bases objetivas, el concepto de bienestar tiene connotaciones subjetivas, particularmente en el sentido de aspiraciones (Arriagada, 2006; Palomar, 2004). Por su parte, la orientación individual habla del bienestar referido a los aspectos de satisfacción con la vida y aspectos positivos del individuo, sirviendo este, a su vez, como un indicador del bienestar social (Temkin \& Del Tronco, 2006). Comparando el enfoque social y psicológico se puede identificar entonces como elemento común para la definición del bienestar al nivel de satisfacción de los individuos y de la sociedad en relación con diferentes áreas de la vida personal y social (relaciones familiares, salud, educación, oportunidades, relaciones interpersonales y oportunidades para el desarrollo pleno).

Concretando en el área psicológica, Diener (1984) ha tenido un papel icónico en el estudio del bienestar, pues a partir de su contribución pionera en la revisión de los estudios sobre la felicidad en 1984 hasta la fecha, el autor ha sistematizado la evidencia empírica y elaboraciones teóricas alrededor de este tópico. Más aún, Diener (1984) ha definido al bienestar como un proceso subjetivo que se refiere a los juicios y evaluaciones que se dan en torno de la satisfacción personal con la vida, así como con el equilibrio de las experiencias afectivas positivas y negativas (Suh \& Oishi, 2002).

Una de las diversas áreas de investigación en las que el bienestar ha despertado gran interés es la de estudios sobre cuidadores de adultos mayores. En ella se considera que el bienestar de los adultos mayores en buena medida depende del bienestar de quien le provee cuidados (Domínguez Guedea \& López Dávalos, 2008), siendo importante estudiarlo no solo por razones válidas para el propio cuidador, sino también por las consecuencias en las tareas del cuidado y en las relaciones entre el adulto mayor y su cuidador (Jolicoeur \& Madden, 2002). Esta es, de hecho, una área de estudios que tiene ya toda una agenda de investigación a nivel internacional, pero dentro de la literatura latinoamericana los trabajos que se reportan al respecto aún son pocos, a pesar del creciente número de adultos mayores y de familiares que asumen la 
carga emocional, física y económica asociadas a la paulatina dependencia funcional que acarrean las enfermedades típicas de la tercera edad.

Con el interés de entender la problemática del cuidador familiar del adulto mayor y de avanzar en el desarrollo de medidas que puedan operacionalizar al bienestar, desde hace algunos años se inició la construcción y validación de una escala de bienestar en cuidadores, dada la escasez de estudios para el desarrollo de instrumentos psicométricos elaborados para este público específico. La autora diseñó y realizó la primera validación exploratoria en población general y en una muestra de cuidadores de adultos mayores de la ciudad de Brasilia (Domínguez Guedea, 2005). Una segunda validación exploratoria la realizó con cuidadores familiares de adultos mayores con diabetes mellitus de la ciudad de Hermosillo, México (López et al., 2010).

El objetivo del presente trabajo es relatar el proceso de diseño, validación exploratoria y confirmatoria de la Escala de Bienestar Subjetivo para Cuidadores Familiares de Adultos Mayores - EBEMS/ CFAM. Para lo anterior, en el siguiente apartado se reseña la fase del diseño y validación en el contexto brasileño de la que originalmente se denominó Escala de Bienestar Subjetivo - EBEMS y posteriormente se relata el estudio de validación exploratoria de la escala en el contexto mexicano, en donde se especificó el nombre de la escala refiriéndola en adelante como Escala de Bienestar Subjetivo en Cuidadores Familiares de Adultos Mayores - EBEMS/CFAM. La metodología y los resultados de este artículo harán referencia al análisis de validación confirmatoria de la EBEMS/CFAM en una muestra de cuidadores familiares de adultos mayores utilizando el Modelamiento Rasch y de ecuaciones estructurales como herramientas estadísticas.

\section{Diseño y validación de la escala en el contexto brasileño}

Como parte de su investigación doctoral, Domínguez Guedea (2005) elaboró la Escala de Bem-estar Subjetivo (EBEMS) con la finalidad de tener una herramienta válida y confiable que pudiera ser utilizada en 
el análisis de un modelo estructural de estrés y bienestar percibidos por familiares cuidadores de adultos mayores dependientes funcionales. Dado que la literatura reporta que el bienestar se integra con el componente cognitivo (satisfacción con la vida) y con el componente afectivo (afectos positivos y afectos negativos), la autora siguió procedimientos específicos para conformar los ítems de cada componente en su escala.

Con relación al componente cognitivo se debe precisar que, aún y cuando en la mayoría de las investigaciones sobre bienestar subjetivo la forma de operacionalizar la satisfacción es con ítems de la vida en general, se consideró necesario crear ítems sobre dominios específicos de la satisfacción con la vida porque existen evidencias apuntando que ese tipo de medida es más sensible a las variables causales (van Praag, Frijters \& Ferrer-i-Carbonell, 2003). Además se ha identificado que los instrumentos sobre satisfacción con la vida en general tienden a producir respuestas vagas (Heylighen \& Bernheim, 2001) y sesgadas por factores situacionales tales como el humor en el momento de responder al cuestionario (Schwarz \& Strack, como se citó en Diener, 2000).

Siendo así, se condujo un primer estudio con la finalidad de identificar temas y categorías sobre dominios específicos de la vida que sirvieran de base para la elaboración de ítems. Se realizó entonces un estudio cualitativo con 21 mujeres y 17 hombres de la población general de la ciudad de Brasilia, seleccionados en forma no probabilística y contactados en lugares públicos. Utilizando la técnica de lluvia de ideas, se solicitaba a cada participante que escribiera todas las ideas que asociara con la satisfacción e insatisfacción en cinco dominios de la vida: familia, amigos, relaciones afectivas, condiciones económicas y creencias religiosas-espirituales. Las respuestas obtenidas fueron tratadas con análisis de contenido de acuerdo a la técnica de Bardin (1977), identificando un grupo de temas y contenidos que fueron útiles para redactar los ítems de la escala correspondientes a la satisfacción con la vida. En la tabla 1 se presenta un resumen de los temas de satisfacción e insatisfacción en los cinco dominios explorados, así como ejemplos de los ítems que se derivaron. El detalle del análisis de contenido del estudio cualitativo está en Domínguez Guedea (2005). 


\section{Tabla 1}

Temas referentes a la satisfacción e insatisfacción en diferentes dominios de la vida

\begin{tabular}{|c|c|c|c|}
\hline $\begin{array}{l}\text { Dominios } \\
\text { de la vida }\end{array}$ & $\begin{array}{l}\text { Temas de } \\
\text { satisfacción }\end{array}$ & $\begin{array}{l}\text { Temas de } \\
\text { insatisfacción }\end{array}$ & $\begin{array}{l}\text { Ejemplos de ítems } \\
\text { derivados de los temas }\end{array}$ \\
\hline Familia & $\begin{array}{l}\text { Unión familiar. } \\
\text { Sentimientos positivos } \\
\text { entre familiares. } \\
\text { Apoyo entre los } \\
\text { miembros de la } \\
\text { familia. } \\
\text { Buena convivencia } \\
\text { familiar. } \\
\text { Logros alcanzados } \\
\text { por la familia. } \\
\text { Actividades de } \\
\text { recreación en la } \\
\text { familia. } \\
\text { Disciplina familiar. }\end{array}$ & $\begin{array}{l}\text { Problemas de } \\
\text { comunicación } \\
\text { en familia. } \\
\text { Falta tiempo } \\
\text { para convivencia } \\
\text { familiar. } \\
\text { Muertes/proble- } \\
\text { mas de salud en } \\
\text { familia. }\end{array}$ & $\begin{array}{l}\text { Yo estoy satisfecho(a) } \\
\text { con... } \\
\text { la convivencia en mi } \\
\text { familia. } \\
\text { la comunicación que } \\
\text { hay en mi familia. } \\
\text { los logros que hemos } \\
\text { alcanzado en mi } \\
\text { familia. } \\
\text { la unión que tenemos } \\
\text { entre los miembros } \\
\text { de la familia. }\end{array}$ \\
\hline $\begin{array}{l}\text { Condi- } \\
\text { ciones } \\
\text { económicas }\end{array}$ & $\begin{array}{l}\text { Dinero para cubrir } \\
\text { gastos diarios. } \\
\text { Dinero para comprar } \\
\text { bienes materiales. } \\
\text { Dinero para cubrir } \\
\text { gastos de recreación. } \\
\text { Dinero para satisfacer } \\
\text { gustos personales. }\end{array}$ & $\begin{array}{l}\text { Alto costo de } \\
\text { vida. } \\
\text { Insatisfacción } \\
\text { con ingreso } \\
\text { familiar. } \\
\text { Limitaciones } \\
\text { para alcanzar } \\
\text { objetivos } \\
\text { personales. }\end{array}$ & $\begin{array}{l}\text { Yo estoy satisfecho(a) } \\
\text { con... } \\
\text { el dinero que tengo } \\
\text { para los gastos diarios. } \\
\text { lo que mi situación } \\
\text { económica me ha } \\
\text { permitido hacer en } \\
\text { la vida. } \\
\text { el dinero que tengo } \\
\text { para comprar } \\
\text { bienes materiales } \\
\text { (ej. muebles, carro, } \\
\text { computadora, etc.). }\end{array}$ \\
\hline
\end{tabular}




\begin{tabular}{|c|c|c|c|}
\hline $\begin{array}{l}\text { Dominios } \\
\text { de la vida }\end{array}$ & $\begin{array}{l}\text { Temas de } \\
\text { satisfacción }\end{array}$ & $\begin{array}{l}\text { Temas de } \\
\text { insatisfacción }\end{array}$ & $\begin{array}{l}\text { Ejemplos de ítems } \\
\text { derivados de los temas }\end{array}$ \\
\hline Amistades & $\begin{array}{l}\text { Apoyo entre amigos. } \\
\text { Sentimientos positivos } \\
\text { en la amistad. } \\
\text { Confianza entre ami- } \\
\text { gos. } \\
\text { Actividades de recrea- } \\
\text { ción compartidas. } \\
\text { Buena convivencia } \\
\text { entre amigos. }\end{array}$ & $\begin{array}{l}\text { Falta de sin- } \\
\text { ceridad entre } \\
\text { amistades. } \\
\text { Conflictos entre } \\
\text { amigos. }\end{array}$ & $\begin{array}{l}\text { Yo estoy satisfecho(a) } \\
\text { con... } \\
\text { las amistades que he } \\
\text { tenido en mi vida. } \\
\text { el apoyo que recibo de } \\
\text { mis amigos. } \\
\text { la sinceridad de mis } \\
\text { amistades. }\end{array}$ \\
\hline $\begin{array}{l}\text { Relaciones } \\
\text { afectivas }\end{array}$ & $\begin{array}{l}\text { Sentimiento de reci- } \\
\text { procidad. } \\
\text { Buena comunicación } \\
\text { en la relación. } \\
\text { Apoyo de pareja. } \\
\text { Convivencia intensa de } \\
\text { pareja. } \\
\text { Formación de familia } \\
\text { de pareja. } \\
\text { Afectividad en la rela- } \\
\text { ción. }\end{array}$ & $\begin{array}{l}\text { Dificultades en } \\
\text { trato y convi- } \\
\text { vencia. } \\
\text { Desacuerdos } \\
\text { sobre la rela- } \\
\text { ción. } \\
\text { Dificultades en } \\
\text { la comunica- } \\
\text { ción. }\end{array}$ & $\begin{array}{l}\text { Yo estoy satisfecho(a) } \\
\text { con... } \\
\text { la conveniencia que } \\
\text { tengo con mi pareja. } \\
\text { el apoyo que he reci- } \\
\text { bido de mi pareja. } \\
\text { lo que compartimos } \\
\text { mi pareja y yo. } \\
\text { el cariño que existe } \\
\text { entre mi pareja y yo. }\end{array}$ \\
\hline $\begin{array}{l}\text { Creencias } \\
\text { religiosas - } \\
\text { espirituales }\end{array}$ & $\begin{array}{l}\text { Fé en Dios. } \\
\text { Concordancia con } \\
\text { costumbres religiosas. } \\
\text { Resultados positi- } \\
\text { vos por práctica de } \\
\text { costumbres religiosas- } \\
\text { espirituales. } \\
\text { Aprendizaje de nuevas } \\
\text { habilidades para convi- } \\
\text { vir mejor. } \\
\text { Creencias religiosas/ } \\
\text { espirituales como guía } \\
\text { de comportamiento. }\end{array}$ & $\begin{array}{l}\text { Incongruencias } \\
\text { en la religión. } \\
\text { Alejamiento } \\
\text { personal de } \\
\text { prácticas y } \\
\text { creencias reli- } \\
\text { giosas-espiri- } \\
\text { tuales. }\end{array}$ & $\begin{array}{l}\text { Yo estoy satisfecho(a) } \\
\text { con... } \\
\text { mi fé. } \\
\text { lo que he aprendido } \\
\text { en mi religión o } \\
\text { grupo espiritual. } \\
\text { los sentimientos que } \\
\text { me transmiten mi } \\
\text { religión-creencias } \\
\text { espirituales. } \\
\text { los resultados de la } \\
\text { religión/creencias } \\
\text { espirituales en mi } \\
\text { vida. }\end{array}$ \\
\hline
\end{tabular}


Por otro lado, los ítems sobre la dimensión afectiva del bienestar subjetivo (afectos positivos y afectos negativos) fueron escogidos de otras escalas de bienestar subjetivo aplicadas en Brasil por Domínguez Guedea (2002) y Albuquerque y Tróccoli (2004). Los ítems integrados en la escala fueron sometidos a un análisis teórico que se conformó por el análisis de jueces —evaluación de la pertinencia de los ítems de acuerdo al constructo que representan- $-\mathrm{y}$ análisis semántico de la comprensión de los ítems, comúnmente llamado de pilotaje. Se hicieron los ajustes correspondientes. La versión del instrumento a validar se conformó por 90 ítems divididos en: a) 15 ítems de sentimientos o emociones positivas y 15 negativas dispuestos en escalas Likert de cinco puntos ( 1 = nada, $5=$ muy fuerte) sobre la intensidad con la cual el respondiente experimentó los sentimientos enlistados, y b) 50 ítems de satisfacción con la vida sobre dominios específicos del constructo: satisfacción con la familia, satisfacción con la vida afectiva, satisfacción con los amigos, satisfacción con las creencias religiosas-morales y satisfacción con las condiciones financieras (10 ítems para cada dominio); además se aplicaron 10 ítems que trataban de la satisfacción con la vida de manera general. Todos los ítems de satisfacción se dispusieron en escala Likert de cinco puntos $(1=$ nada satisfecho/a, 5 = muy satisfecho/a).

Esa versión se piloteó y, después de los debidos ajustes, se aplicó a 507 personas de la ciudad de Brasilia. La colecta de datos se hizo respetando cuotas equivalentes por sexo y grupos de edad (de 18 a 84 ańos) de la población general, seleccionados en forma no probabilística y contactados en lugares públicos. Se aplicaron procedimientos de verificación de presupuestos multivariados para asegurar que el conjunto de datos mostraran atributos de normalidad, linealidad, ausencia de casos discrepantes multivariados y de multi-colinealidad, siendo necesario para eso retirar ítems y observaciones que desviaran a las distribuciones de las exigencias básicas para aplicar análisis multivariados. De esta manera, el banco final de datos se conformó por 451 participantes y un total de 74 variables.

Con ese conjunto de datos se determinaron las propiedades psicométricas de la EBEMS/CFAM aplicándose análisis de factorabilidad, análisis paralelo, análisis factorial con rotación oblimin (dadas las correlaciones entre factores $\geq .30$ ) y análisis de confiabilidad. Se obtuvo 
una solución de cinco factores con 43 ítems válidos y confiables. En la segunda columna de la tabla 2 se resumen las propiedades psicométricas obtenidas en esta aplicación.

Después de su aplicación en población general, fue necesario producir una versión reducida de la escala para contar con una herramienta compacta que pudiera ser aplicada a una muestra de cuidadores, junto con un conjunto de instrumentos de medida. La versión reducida se hizo seleccionando los seis ítems con mayores cargas factoriales de cada factor, contando con 30 ítems (cargas factoriales $\geq .45$ ) que en conjunto explicaron el 53\% de la varianza del bienestar subjetivo, siendo los mismos factores que los hallados en la versión extensa, con alfas $\geq .84$. Esta versión reducida se aplicó a una muestra de 187 familiares cuidadores de adultos mayores dependientes funcionales, también de Brasilia. En esta aplicación se disminuyó a cuatro puntos la escala de respuesta para evitar la eventual sobrecarga de respuestas en el punto intermedio. Se repitió la secuencia de análisis de validación en la muestra de cuidadores, encontrando que con 28 ítems (cargas factoriales $\geq .45$ ) se explicaba el $54.8 \%$ de la varianza del constructo (ver tercera columna de tabla 2).

Enseguida se realizaron Análisis Factoriales Confirmatorios (AFC) de los datos, encontrando que los cuatro factores representaron adecuadamente a la variable latente bienestar subjetivo con coeficientes de regresión estandarizados significativos $p \geq .59$. Los índices de ajuste del modelo de medida fueron: $\chi^{2}(2,187)=3.93, p \geq .05 ; \chi^{2} / d f=1.96$; $\mathrm{GFI}=.99, \mathrm{NFI}=.98, \mathrm{CFI}=.99 ; \mathrm{RMSEA}=.07$ (intervalo de confianza de $95 \%=.000$ a 0.18 ). De acuerdo a los resultados, el bienestar subjetivo tiene una relación causal positiva con los afectos positivos y la satisfacción con el dinero, así como una relación causal negativa con los afectos negativos y con la insatisfacción con las relaciones interpersonales (Domínguez Guedea, 2005). De esta forma, la EBEMS/CFAM mostró resultados consistentes en términos conceptuales y empíricos, permitiendo afirmar la confiabilidad y validez exploratoria entre cuidadores familiares de adultos mayores en Brasilia, pero se aclara que, aún y cuando se aplicaron AFC, no se asumió en ese momento del proceso la validez confirmatoria de la escala, optando observar su comportamiento en otras muestras de cuidadores. 
Escala de Bienestar Subjetivo en Cuidadores Familiares de Adultos... / Domínguez Guedea et al.

\section{Tabla 2}

Comparación de caracteristicas de la EBEMS/CFAM validada en tres muestras independientes

\begin{tabular}{|c|c|c|c|}
\hline Características & $\begin{array}{l}\text { Población general } \\
\text { brasileña }\end{array}$ & $\begin{array}{l}\text { Cuidadores } \\
\text { brasileños }\end{array}$ & $\begin{array}{l}\text { Cuidadores } \\
\text { mexicanos }\end{array}$ \\
\hline Ítems & 74 & 30 & 22 \\
\hline Casos analizados & 451 & 187 & 139 \\
\hline Varianza explicada & $48 \%$ & $54.8 \%$ & $55 \%$ \\
\hline $\begin{array}{l}\text { Ítems retenidos } \\
\text { Rango de cargas } \\
\text { factoriales }\end{array}$ & $\begin{array}{l}43 \\
\text { de } .43 \text { a } .82\end{array}$ & $\begin{array}{l}28 \\
\text { de } .34 \text { a } .88\end{array}$ & $\begin{array}{l}20 \\
\text { de } .35 \text { a } .91\end{array}$ \\
\hline Factor 1 & $\begin{array}{l}\text { Afectos positivos } \\
(12 \text { ítems; alfa }=.89)\end{array}$ & $\begin{array}{l}\text { Afectos negativos } \\
(6 \text { ítems; alfa }=.80)\end{array}$ & $\begin{array}{l}\text { Satisfacciones } \\
\text { materiales } \\
(6 \text { ítems; alfa = } \\
.91)\end{array}$ \\
\hline Factor 2 & $\begin{array}{l}\text { Afectos negativos } \\
(13 \text { ítems; alfa }=.90)\end{array}$ & $\begin{array}{l}\text { Satisfacción / creen- } \\
\text { cias religiosas } \\
(6 \text { ítems; alfa }=.89)\end{array}$ & $\begin{array}{l}\text { Satisfacciones } \\
\text { personales } \\
(6 \text { ítems; alfa = } \\
.84)\end{array}$ \\
\hline Factor 3 & $\begin{array}{l}\text { Satisfacción / condi- } \\
\text { ciones económicas } \\
(6 \text { ítems; alfa }=.90)\end{array}$ & $\begin{array}{l}\text { Satisfacción / condi- } \\
\text { ciones económicas } \\
(6 \text { ítems; alfa }=.90)\end{array}$ & $\begin{array}{l}\text { Afectos } \\
\text { negativos } \\
(4 \text { items; alfa = } \\
.82)\end{array}$ \\
\hline Factor 4 & $\begin{array}{l}\text { Satisfacción / relacio- } \\
\text { nes interpersonales } \\
(6 \text { ítems; alfa }=.84)\end{array}$ & $\begin{array}{l}\text { Afectos positivos } \\
\text { (5 ítems; alfa }=.77 \text { ) }\end{array}$ & $\begin{array}{l}\text { Afectos } \\
\text { positivos } \\
(8 \text { ítems; alfa = } \\
.74)\end{array}$ \\
\hline Factor 5 & $\begin{array}{l}\text { Satisfacción / creen- } \\
\text { cias religiosas } \\
(6 \text { ítems; alfa }=.88 \text { ) }\end{array}$ & \multicolumn{2}{|c|}{$\begin{array}{l}\text { Satisfacción / relacio- } \\
\text { nes interpersonales }(5 \text {--- } \\
\text { ítems; alfa }=.79)\end{array}$} \\
\hline
\end{tabular}




\section{Validación y utilidad de la escala en el contexto mexicano}

Posteriormente surgió la necesidad de medir el bienestar subjetivo entre cuidadores familiares de adultos mayores mexicanos de Hermosillo, Sonora con diabetes mellitus, dado que esta enfermedad es una de las grandes preocupaciones en salud pública a nivel nacional y estatal, con índices de morbi-mortalidad acentuados en la tercera edad. Por esa razón, Domínguez-Guedea y colaboradores $(2006,2007)$ iniciaron un conjunto de estudios sobre los factores asociados a la adherencia terapéutica entre diabéticos adultos mayores y en los cuales invariablemente surgió la importancia del bienestar del cuidador familiar.

Dado que la EBEMS/CFAM fue validada en cuidadores familiares brasileños, su nuevo uso exigía la validación en el actual contexto cultural de referencia. Aunque en México hay instrumentos con propiedades psicométricas robustas para medir el bienestar (e. g. Escala Multidimensional para la Medición del Bienestar Subjetivo de Anguas-Plata \& Reyes-Lagunes, 1999), su validación fue realizada con la población general del Distrito Federal o del noroeste de México (Vera \& Tánori, 2002). Ante esta situación, Domínguez Guedea y colaboradores validaron la EBEM/CFAM en el contexto de Hermosillo, ciudad al noroeste de México (López et al., 2010). Se efectuó la traducción/re-traducción del instrumento, mismo que fue piloteado y ajustado semánticamente, y en su validación se siguió la secuencia que incluye el análisis de presupuestos multivariados, de factorabilidad, análisis paralelo, análisis factorial con rotación oblimin y análisis de confiabilidad. En la tabla 2 (cuarta columna) se resumen las características psicométricas de la escala en cuidadores mexicanos.

Un nuevo conjunto de técnicas para probar las propiedades de la EBEMS/CFAM consistió en su análisis bajo el Modelamiento Rasch y el Análisis Factorial Confirmatorio. 


\section{Método}

\section{Participantes}

La muestra se constituyó de 142 cuidadores familiares de adultos mayores con diabetes mellitus tipo 2 , siendo $81.2 \%$ mujeres y el resto hombres; las edades fluctuaron entre los 18 y 90 años $(M=47.17 ; D E$ = 16.65). En cuanto al parentesco con el paciente, $51 \%$ de los cuidadores eran los cónyuges, 39\% eran hijas(os) y 10\% eran hermanas(os), nietas(os) o parientes políticos del adulto mayor. La media de edad de los adultos mayores receptores de los cuidados fue de 67 años $(\sigma=7.2)$. Además de vivir en Hermosillo, Sonora, los participantes debían ser cuidadores primarios de un familiar adulto mayor que necesitara ayuda para realizar al menos una de las actividades de la vida diaria y compartir la misma residencia con el adulto mayor, o bien que el cuidador lo visitara por lo menos una vez a la semana.

\section{Instrumentos}

Se utilizó la Escala de Bienestar Subjetivo para Cuidadores Familiares de Adultos Mayores (EBEMS/CFAM) cuyos datos de validación exploratoria en cuidadores mexicanos están resumidos en la tabla 2 . En la primera parte del instrumento se exploran los afectos positivos y negativos, mediante una lista de sentimientos y emociones ante los cuales se pregunta la intensidad con la que fueron experimentados en el último mes; las respuestas a los reactivos son de tipo Likert $(1=$ no sentí, 2 = sentí un poco, 3 = sentí regular, 4 = sentí mucho). La segunda parte de la escala explora la satisfacción con la vida constando de 15 ítems con opciones de respuesta de tipo Likert ( 1 = nada satisfecho/a, 2 = poco satisfecho/a, 3 = satisfecho/a, 4 = muy satisfecho/a).

Para colectar los datos de identificación, la primera autora de este trabajo elaboró un cuestionario para los fines del estudio, en el cual se abordan datos sociales, económicos y demográficos del cuidador y del adulto mayor que recibe los cuidados. 


\section{Procedimiento}

Para identificar a los participantes se contactó a pacientes que forman parte de Grupos de Ayuda Mutua (GAM) para pacientes diabéticos e hipertensos de cuatro centros urbanos de salud. Además se realizaron visitas domiciliarias en el radio de atención de los centros de salud, identificando a cuidadores y a quienes cumplían con los criterios de inclusión. Se solicitaba la participación voluntaria. Para la colecta la información fueron necesarias de tres a cuatro visitas por caso (con un promedio de una hora de duración en cada visita) ya que, además de la EBEMS/CFAM, se levantaba otro conjunto de instrumentos. Los datos fueron colectados combinando las estrategias de entrevista y auto-administración de instrumentos, contando siempre con el auxilio del encuestador. Se consultó la participación voluntaria mediante una carta de Consentimiento Informado. El proyecto general del cual se deriva este estudio fue evaluado por la Academia de Psicología Clínica y de la Salud de la Universidad de Sonora que, entre otros aspectos, lo analizó con relación a consideraciones éticas al no existir un comité especializado en dicha Universidad. Asimismo, el proyecto recibió la aprobación del Departamento de Investigación de la Dirección General de Enseñanza y Calidad de la Secretaría de Salud de Sonora.

\section{Resultados}

Como primer paso se examinó el cumplimiento de presupuestos multivariados en el banco de datos, encontrando que no había ítems cuyas distribuciones mostraran valores de asimetría $\geq 1$, así como tampoco existían violaciones a la linealidad (verificadas con scatterplots) entre pares de variables; se asumió la ausencia de multicolinealidad dados los valores de tolerancia $\leq .300 \mathrm{y}$ referente a los casos discrepantes multivariados (outliers), se encontraron y eliminaron tres casos extremos al obtener la distancia de Mahalanobis y compararla con el valor crítico de $\chi^{2}=55.47, p \geq .01$. Realizadas estas pruebas preliminares, se continuó con los análisis de validación. 
Los resultados previamente relatados sobre la EBEMS/CFAM demostraron que el constructo del bienestar no es unidimensional, pero sus cuatro factores sí lo son (satisfacciones materiales, satisfacciones personales, afectos negativos y afectos positivos). De esta manera, se aplicaron análisis de Modelamiento Rasch al conjunto de ítems que conforman cada uno de los factores del bienestar. En la tabla 3 se muestran los análisis de cada factor.

\section{Tabla 3}

Análisis Rasch para cada uno de los factores de la EBEMS validada en cuidadores de adultos mayores en contexto mexicano

\begin{tabular}{|c|c|c|c|c|c|c|c|c|}
\hline $\begin{array}{l}\text { Factor de } \\
\text { la EBEMS/ } \\
\text { CFAM }\end{array}$ & $\mathrm{em}$ & $\begin{array}{l}\text { Medida } \\
\text { lógitos }\end{array}$ & $\begin{array}{c}\text { Error } \\
\text { están- } \\
\text { dar }\end{array}$ & $\begin{array}{l}\text { INFIT } \\
\text { MNSQ }\end{array}$ & $\begin{array}{l}\text { INFIT } \\
\text { ZSTD }\end{array}$ & $\begin{array}{l}\text { OUTFI' }^{2} \\
\text { MNSQ }\end{array}$ & $\begin{array}{l}\text { OUTFIT } \\
\text { ZSTD }\end{array}$ & DIS \\
\hline \multirow{6}{*}{$\begin{array}{l}\text { Satisfacciones } \\
\text { materiales } \\
\text { Conf }=.94 \\
\text { RMSE }=.18\end{array}$} & S4 & .2 & 0.17 & 1.1 & 0.75 & 1.1 & 0.71 & 0.82 \\
\hline & S7 & -0.02 & 0.18 & 0.78 & -1.8 & 0.74 & -2.04 & 0.87 \\
\hline & S8 & 1.3 & 0.17 & 0.98 & -0.08 & 0.94 & -0.34 & 0.84 \\
\hline & S9 & -0.62 & 0.2 & 0.9 & -0.72 & 0.84 & -1.05 & 0.84 \\
\hline & S11 & 0.24 & 0.17 & 1.02 & 0.22 & 0.98 & -0.11 & 0.82 \\
\hline & S14 & -1.1 & 0.19 & 1.12 & 0.94 & 1.07 & 0.53 & 0.8 \\
\hline \multirow{6}{*}{$\begin{array}{l}\text { Satisfacciones } \\
\text { personales } \\
\text { Conf }=.62 \\
\text { RMSE }=.19\end{array}$} & $S$ & s & 0 & & 0.74 & 1.1 & 0.1 & 0.7 \\
\hline & S5 & -0.33 & 0.19 & 0.98 & -0.09 & 0.98 & -0.09 & 0.72 \\
\hline & S6 & -0.29 & 0.17 & 1 . & .98 & 1. & 223 & 0.64 \\
\hline & S12 & 0.34 & 0.16 & 1.00 & 0.02 & 1.00 & 0.04 & 0.73 \\
\hline & S13 & 0.46 & 0.1 & 0.96 & -0.26 & 0.97 & -0.18 & 0.76 \\
\hline & S15 & -0.01 & 0.19 & 0.67 & -2.31 & 0.61 & -2.76 & 0.8 \\
\hline \multirow{4}{*}{$\begin{array}{l}\text { Afectos } \\
\text { negativos } \\
\text { Conf }=.93 \\
\text { RMSE }=.15\end{array}$} & ANG & -0.83 & 0.1 & 0.96 & -0.28 & 0.9 & -0.68 & 0.82 \\
\hline & SUFRI & 0.51 & 0.14 & 0.79 & -1.66 & 0.79 & -1.51 & 0.81 \\
\hline & TRIST & -0.27 & 0.14 & 0.97 & -0.19 & 1.00 & 0.04 & 0.81 \\
\hline & DESÁN & 0.59 & 0.15 & 1.25 & 1.89 & 1.24 & 1.79 & 0.74 \\
\hline
\end{tabular}




\begin{tabular}{llrrrrrrr}
\hline $\begin{array}{l}\text { Factor de } \\
\text { la EBEMS/ } \\
\text { CFAM }\end{array}$ & Ítem & $\begin{array}{l}\text { Medida } \\
\text { lógitos }\end{array}$ & $\begin{array}{l}\text { Error } \\
\text { están- } \\
\text { dar }\end{array}$ & $\begin{array}{l}\text { INFIT INFIT OUTFIT OUTFIT Ptbis } \\
\text { MNSQ ZSTD MNSQ }\end{array}$ & ZSTD & \\
\hline $\begin{array}{l}\text { Afectos posi- } \\
\text { tivos }\end{array}$ & ENTUS & 0.8 & 0.14 & 1.03 & 0.24 & 1.01 & 0.1 & 0.77 \\
$\begin{array}{l}\text { Conf }=.90 \\
\text { RMSE }=.15\end{array}$ & GUSTO & -0.37 & 0.16 & 0.86 & -1.08 & 0.88 & -0.92 & 0.75 \\
& CONFI & -0.04 & 0.15 & 0.98 & -0.14 & 0.99 & -0.02 & 0.72 \\
\hline
\end{tabular}

Nota $:$ Conf = confiabilidad; RMSE = error cuadrático medio.

Bajo la lógica de estos análisis, la validez de constructo está dada por los resultados de los Índices INFIT y OUTFIT, cuyos valores en medias cuadráticas (MNSQ) deben mantenerse en el rango de .50 a 1.50 en escalas de percepción - siendo el valor 1 el indicador de ajuste perfecto entre los datos y el modelo- y en valores estandarizados (ZSTD) los valores deben oscilar entre $-/+2$ (González-Montesinos, 2008). Los resultados de la tabla 3 confirman la validez de los ítems en sus dimensiones/factores correspondientes, pues no presentan desajustes en relación con el modelo probabilístico. El único caso que se distanció más del criterio establecido fue el ítem S15 (satisfacción con los resultados de la religión/creencias espirituales en mi vida), que presentó valores de INFIT y OUTFIT de .67 y, aunque no sobrepasó el criterio establecido, se optó retirarlo de la dimensión para cohesionar mas la escala.

Los valores de las correlaciones punto biserial (Ptbis) $\geq .7$ confirman la unidimensionalidad de cada factor del bienestar y la pertenencia de cada ítem al factor; asimismo, los índices de confiabilidad indican la consistencia interna de los cuatro factores. Además, los valores de RMSE $\leq .19$ indican que los errores sistemático y aleatorio en los datos no son preocupantes, pues entre más baja sea su magnitud, más próximos están los parámetros a los valores verdaderos. De esta forma, la contribución que ofrecieron los análisis Rasch al proceso de validación de la EBEMS/CFAM fue la posibilidad de obtener medidas independientes de la variación del instrumento de los de participantes, abonando a la robustez de la medida. 
Con este banco de datos se aplicaron también Análisis Factoriales Confirmatorios reafirmando que los cuatro factores representan adecuadamente a la variable latente bienestar subjetivo. Los índices de ajuste del modelo de medida fueron: $\chi^{2}(1 ; 139)=.94, p \geq .05 ; \chi^{2} / d f=$ $.94 ; \mathrm{GFI}=.99, \mathrm{NFI}=.99, \mathrm{CFI}=1.00 ; \mathrm{AGFI}=.96 ; \mathrm{RMSEA}=.000$ (intervalo de confianza de $90 \%=.000$ a 0.22 ). Así, se demostró que el bienestar subjetivo tiene una relación causal positiva y significativa con los afectos positivos $(\beta=.83)$, con satisfacciones personales $(\beta=$ .49), satisfacciones materiales $(\beta=.56)$, y una relación causal negativa y significativa con los afectos negativos $(\beta=-.59)$. Se observó también la covarianza de .31 entre los errores de medida de los factores satisfacciones personales y satisfacciones materiales. La magnitud de los pesos factoriales bajo la lógica de las ecuaciones estructurales señala una fuerte relación de la variable latente (bienestar subjetivo) con sus indicadores (afectos positivos, afectos negativos, satisfacciones personales y satisfacciones materiales).

\section{Comentario}

El análisis del bienestar de los cuidadores familiares de adultos mayores tal vez sea uno de los temas que más interés ha generado en la literatura sobre gerontología social (Deimling et al., 2001), dinamizando numerosas investigaciones que analizan los predictores del bienestar así como su contribución a los buenos cuidados hacia el adulto mayor. No obstante, aún y cuando la mayoría de las investigaciones argumentan la importancia del bienestar, son pocas las que efectivamente lo miden, siendo común que en su lugar sean evaluadas variables tales como depresión y ansiedad (e. g. Berger et al., 2005; Lawrence, Tennstedt \& Assmann, 1998; Martire, Franks \& Atienza, 1997; Son et al., 2007), probablemente debido a la suposición de que bajos niveles de depresión equivalen a altos niveles de bienestar (Gibson, 2003). Se ha evaluado también sobrecarga asumiendo que esta corresponde a un desgaste en el bienestar, sin embargo ambos atributos no son necesariamente opuestos 
pues hay cuidadores que pueden sentirse sobrecargados pero satisfechos con su vida y mantienen niveles razonables de bienestar (Chapell \& Reid, 2002).

Siendo así, en esta área de investigación el constructo de bienestar frecuentemente ha sido tratado de manera imprecisa, considerándolo como un estado general que requiere ser traducido de indicadores de connotación negativa, lo cual es injustificado, pues el bienestar tiene componentes claramente definidos por lo que no debe ser tratado de manera ambigua ni asumido como el opuesto a una diversidad de sentimientos que el cuidador pueda tener. Al tener una mirada parcial es difícil "explicar cómo algunas personas hacen frente a la adversidad con un temple y una entereza que asombra a sus congéneres y provocan profunda admiración" (Cuadra \& Florenzano, 2003, p. 93). Acorde con la anterior afirmación, Hochschild (2003) señala que el bienestar proporciona el medio para producir y manejar emociones que un sujeto siente más apropiadas para la identidad social en la que está inmerso.

Por otro lado, la práctica de combinar en los estudios sobre cuidadores los componentes del bienestar satisfacción con la vida o afectos positivos, con medidas de depresión o ansiedad (e. g. Arango-Lasprilla, 2009; Gignac \& Gottlieb, 1996; Gitlin, Winter, Dennis \& Hauck, 2006; Levesque, Cossetle \& Laurin, 1995; Stephens \& Townsend, 1997), dificulta extraer resultados específicos del bienestar. El bienestar subjetivo funge como un resumen psicológico de la calidad de vida de un individuo en la sociedad y aunque otros conceptos de la psicología social se relacionan con la calidad de vida (e. g. autoestima, depresión y locus de control), únicamente el componente cognitivo del bienestar, la satisfacción de vida, implica un resultado conclusivo en términos de consecuencias para el individuo (Andrews \& Robinson, 1991). Por otro lado, el componente emocional, equilibrio de los afectos, refiere a la noción de que las crisis son inevitables y necesarias para el desarrollo humano, de forma que "el bienestar psicológico no es simplemente la vida feliz 'per se' sino los procesos del vivir con sus mezclas de sabores dulces y amargos" (Cuadra \& Florenzano, 2003, p. 93). 
En suma, aunque el bienestar esté presente en el discurso de la mayoría de los estudios con cuidadores, el conocimiento sobre su medida ha sido limitado (Chapell \& Reid, 2002). Buscando atenuar esa laguna, el objetivo de este trabajo fue relatar el proceso de diseño y validación de una escala de bienestar específica para cuidadores familiares de adultos mayores. La Escala de Bienestar Subjetivo en Cuidadores Familiares de Adultos Mayores (EBEMS/CFAM) ha demostrado propiedades psicométricas robustas tanto en el contexto brasileño como en el mexicano y específicamente en cuidadores familiares de adultos mayores. En todas las muestras en las cuales la escala fue aplicada, los porcentajes de varianza explicada fueron muy satisfactorios ( $\geq 48 \%$ ), indicando que el instrumento realmente mide lo que pretende medir. Como otro aspecto relacionado con la validez de la medida, se tiene que las saturaciones factoriales alcanzadas en cada uno de los ítems que constituyen la escala fueron $\geq .34$, refiriendo la precisión de los reactivos para medir los diferentes componentes del bienestar.

En términos de la confiabilidad, evaluada mediante el método de consistencia interna, se tiene que en los tres estudios de validación se obtuvieron valores alfa de Cronbach $\geq .77$, revelando suficiente cohesión entre el conjunto de ítems que integran cada factor. Los resultados de los análisis Rasch afirman la validez de la EBEMS/CFAM en cada uno de los factores de la medida. Finalmente, los Análisis Factoriales Confirmatorios realizados confirman que los cuatro factores representan adecuadamente a la variable latente bienestar subjetivo, teniendo una relación causal positiva y significativa con los afectos positivos, con satisfacciones personales y satisfacciones materiales, así como una relación causal negativa y significativa con los afectos negativos.

Así, partiendo de una propuesta inicial conformada por 90 ítems, la EBEMS/CAM consta actualmente de 20 ítems. La escala mostró resultados consistentes en términos conceptuales y empíricos, permitiendo afirmar la confiabilidad y validez, ya no solo en términos exploratorios sino también confirmatorios. La consistencia de los resultados encontrados en tres muestras (población abierta y de cuidadores familiares brasileños y mexicanos) y con distintas aproximaciones analíticas 
fortalecen la capacidad de la EBEMS/CFAM para medir de manera válida y confiable el atributo de bienestar, confirma la estructura teórica de este constructo y ofrece un recurso psicométrico que permite comprender mejor la situación del cuidador familiar. Como fue indicado anteriormente, el análisis del bienestar tiene su utilidad multidisciplinar comprobada, siendo un constructo relevante no solo en estudios psicológicos, sino también económicos, sociológicos y geriátricos. La elección entre diversas medidas de bienestar debe ser cuidadosa, ya que esto tiene importantes implicaciones a la hora de diseñar e implementar políticas públicas (Angner, 2011).

\section{Referencias}

Albuquerque, A. S. \& Tróccoli, B. T. (2004). Desenvolvimento de uma escala de bem-estar subjetivo. Psicologia: Teoria e pesquis, 20(2), 153-164.

Andrews, F. M. \& Robinson, J. P. (1991). Measures of subjective wellbeing. En J. P. Robinson, P. R. Shaver \& L. S. Wrightsman (Eds.), Measures of personality and social psychological attitudes (pp. 61-114). San Diego, CA: Academic Press.

Angner, E. (2011). Are subjective measures of well-being 'direct'? Australasian Journal of Philosophy, 89(1), 115-130.

Anguas-Plata, A. M. \& Reyes-Lagunes, I. (1999). El significado del bienestar subjetivo: su valoración en México. La Psicología Social en México, 7, 7-11.

Arango-Lasprilla, J. C., Moreno, A., Rogers, H. \& Francis, K. (2009). The effect of dementia patient's physical, cognitive, and emotional/behavioral problems on caregiver well-being: Findings from a Spanish-speaking sample from Colombia, South America. American Journal of Alzheimer's Disease \& Other Dementias, 24(5), 384-395.

Arriagada, I. (2006). Cambios de las politicas sociales: politicas de género y familia. Santiago de Chile: CEPAL. 
Bardin, L. (1977). Análise de conteúdo. Lisboa: Edições 70.

Berger et al. (2005). Longitudinal study on the relationship between symptomatology of dementia and levels of subjective burden and depression among family caregivers in memory clinic patients. Journal of Geriatric Psychiatry and Neurology, 18(3), 119-128.

Cuadra, H. \& Florenzano, R. (2003). El bienestar subjetivo: hacia una psicología positiva. Revista de Psicología de la Universidad de Chile, 12(1), 83-96.

Diener, E. (1984). Subjective well-being. Psychological Bulletin, 95, $542-575$.

Diener, E. (2000). Subjective well-being. The science of happiness and a proposal for a National Index. American Psychological Association, 55(1), 34-43.

Domínguez-Guedea, M. (2002). Analise do bem-estar subjetivo em idosos (Tesis de maestría inédita). Universidade da Paraíba, João Pessoa, Brasil.

Domínguez-Guedea, M. (2005). Modelo de estrés y bienestar subjetivo en cuidadores de familiares de adultos mayores dependientes funcionales (Tesis de doctorado inédita). Universidade de Brasília, Brasil.

Domínguez-Guedea, M. (2006). Análisis de factores familiares que promueven la adherencia terapéutica entre adultos mayores con diabetes mellitus. Manuscrito inédito, Universidad de Sonora, Hermosillo, México.

Domínguez-Guedea, M. (2007). Evaluación de características psicosociales del paciente diabético adulto mayor para el diseño de programas de apoyo a la adherencia terapéutica y el control gliémico (Informe de investigación inédito). México: Fondo Sectorial de Investigación en Salud y Seguridad Social SSA/IMSS/ISSTE-CONACYT.

Domínguez-Guedea, M. \& López-Dávalos, G. (2008). Bienestar de cuidadores familiares de adultos mayores. Sonárida, 12(26) 13-16.

Draibe, S. \& Riesco, M. (2009). El estado de bienestar social en América Latina, una nueva estrategia de desarrollo. Madrid: Fundación Carolina. Recuperado de http://www.fundacioncarolina.es/ esES/publicaciones/documentostrabajo/Documents/ DT31 pdf 
Gitlin, L. N., Winter, L., Dennis, M. P. \& Hauck, W. W. (2006). Assessing perceived change in the well-being of family caregivers: Psychometric properties of the Perceived Change Index and Response Patterns. American Journal of Alzheimer's Disease \& Other Dementias, 21(5), 304-311.

González-Montesinos, M. (2008a). Programa de asesoría y actualización en psicometría. Notas para Módulo 3. Elementos de Teoría Clásica de la Medida. Análisis de Dimensionalidad. Recuperado de http:// metcuantus.org/wp/wp-content/uploads/2008/08/materialm3-comw.doc

Heylighen, F. \& Bernheim, J. (2001). Measuring global progress through subjective well-being. Trabajo presentado en la III Conference of the ISQOLS, Universidad de Girona, España.

Hochschild, A. R. (2003). The commercialization of intimate life: Notes from home and work. Berkeley, CA: University of California.

Jolicoeur, P. M. \& Madden, T. (2002). The good daughters: Acculturation and caregiving among Mexican-American women. Journal of Aging Studies, 16, 107-120.

Levesque, L., Cossetle, S. \& Laurin, L. (1995). A multidimensional examination of the psychological and social well-being of caregivers of a dementia relative. Research on Aging, 17(3), 332-360.

López-Dávalos, G., Domínguez-Guedea, M., Ochoa, P., Montiel, M., Laborín, J., Domínguez, R. et al. (2010). Validación de la Escala de Bienestar Subjetivo (EBEMS) en cuidadores familiares de adultos mayores. Psicología y Salud, 20(1), 13-22.

Ochoa-Marcobich, M. P. \& López-Davalos, G. (2008). Validación de la Escala de Bienestar Subjetivo en cuidadores familiares de adultos mayores con Diabetes Mellitus tipo II (Tesis de licenciatura inédita). Universidad de Sonora, Hermosillo, México.

Palomar, L. J. (2004). Pobreza, recursos psicológicos y bienestar subjetivo (Documento de Investigación No. 3). México: Instituto de Investigaciones sobre Desarrollo Sustentable y Equidad Social, Universidad Iberoamericana. Recuperado de http://www.uia. $\mathrm{mx} / \mathrm{campus} /$ publicaciones/IIDSES/pdf/investigacion/idses3.pdf 
Temkin, B. \& Del Tronco, J. (2006). Desarrollo humano, bienestar subjetivo y democracia: confirmaciones, sorpresas e interrogantes. Revista Mexicana de Sociología, 68(4), 731-760.

Son, J., Erno, A., Shea, D. G., Femia, E. E., Zarit, S. H. \& Stephens, M. A. (2007). The caregiver stress process and health outcomes. Journal of Aging and Health, 19(6), 871-887.

Suh, E. M. \& Oishi, S. (2002). Subjective well-being across cultures. En W. J. Lonner, D. L. Dinnel, S. A. Hayes \& D. N. Sattler (Eds.), Online readings in psychology and culture. Recuperado de http://www.ac.wwu.edu/ culture/readings.htm

Van Praag, B. M., Frijters, P. \& Ferrer-i-Carbonell, A. (2003). The anatomy of subjective well-being. Journal of Economic Behavior and Organization, 51, 29-49.

Vera N. J. A. \& Tánori A. B. C. (2002). Propiedades psicométricas de un instrumento para medir bienestar subjetivo en la población mexicana. Apuntes de Psicología, 20(1), 5-23.

Recibido: 17 de febrero, 2011 Aceptado: 30 de junio, 2011 